\title{
Noisy Reverberation Suppression Using AdaBoost Based EMD in Underwater Scenario
}

\author{
Kusma Kumari Cheepurupalli and Raja Rajeswari Konduri \\ Department of ECE, College of Engineering, Andhra University, Visakhapatnam, India \\ Correspondence should be addressed to Kusma Kumari Cheepurupalli; chkusumasrinivas@gmail.com
}

Received 23 August 2013; Revised 10 December 2013; Accepted 19 December 2013; Published 4 February 2014

Academic Editor: Heinrich Hühnerfuss

Copyright ( 2014 K. K. Cheepurupalli and R. R. Konduri. This is an open access article distributed under the Creative Commons Attribution License, which permits unrestricted use, distribution, and reproduction in any medium, provided the original work is properly cited.

\begin{abstract}
Reverberation suppression is a crucial problem in sonar communications. If the acoustic signal is radiated in the water as medium then the degradation is caused due to the reflection coming from surface, bottom, and volume of water. This paper presents a novel signal processing scheme that offers an improved solution in reducing the effect of interference caused due to reverberation. It is based on the combination of empirical mode decomposition (EMD) and adaptive boosting (AdaBoost) techniques. AdaBoost based EMD filtering technique is used for reverberation corrupted chirp signal to decrease the noisy components present in the received signal. An improvement in the probability of detection is achieved using the proposed algorithm. The simulation results are obtained for various reverberation times at various SNR levels.
\end{abstract}

\section{Introduction}

Underwater acoustic is the study of propagation of sound in water. The water may be in the ocean, a lake, or a tank. Typical frequencies used in underwater environment lie between $10 \mathrm{~Hz}$ to $1 \mathrm{MHz}$. The propagation of sound in underwater environment is affected by reverberation caused due to scatters from rough boundaries and due to fish and other biota (total collection of organisms). For an acoustic signal to be detected easily, it must exceed the reverberation level and background noise level $[1,2]$. Various methods have been published in the literature to reduce the effect of noise. Among them, empirical mode decomposition [3] is considered as one of the best methods for noise reduction application.

The motivation behind the use of EMD as a filtering technique is that it is completely adaptive and data driven method that operates on nonlinear and nonstationary data which are generally encountered in the real environment. The main advantage of EMD is that it does not depend on the filter orders like linear and adaptive filters $[4,5]$ and also does not require any basic or prior function as in the case like wavelets $[6,7]$. EMD also exhibits stable performance along with moderate speed and less complexity.
In our previous work $[8,9]$ the noise reduction problem due to interference signals was addressed using EMD as a filtering technique in which the selection of intrinsic mode functions (IMFs) which are used for signal reconstruction, was done manually. In this paper we propose the use of adaptive boosting technique [10] to adaptively select the IMFs produced by EMD.

Sections 2 and 3 describe the brief introduction on EMD and AdaBoost techniques and Section 4 presents the description of new structure of the AdaBoost based EMD filtering technique by considering a chirp signal $s(t)$ as the original input signal which is corrupted by both reverberation (nonstationary and colored) and noise (additive white Gaussian noise) as $n(t)$. The superior performance of the new algorithm is demonstrated using a set of simulation results.

\section{Empirical Mode Decomposition}

Empirical Mode Decomposition (EMD) is relatively unconventional method in Signal Processing proposed by Huang et al. [3]. The decomposition method used in the EMD is called as "Sifting" process. The introduction of these instantaneous frequencies for complicated data sets is used to eliminate the spurious harmonics that may present in nonlinear and 
nonstationary signals. The algorithm is described in the following steps.

The input signal applied to EMD is $x(t)=s(t)+n(t)$.

(1) Identify all extrema of input signal $x(t)$.

(2) Generate the upper envelope $u(t)$ and the lower envelope $l(t)$ of extrema and calculate the mean envelope as

$$
m(t)=\frac{u(t)+l(t)}{2}
$$

(3) Subtract $m(t)$ from $x(t)$ to generate the detail

$$
d(t)=x(t)-m(t) .
$$

(4) Update $x(t)$ using $d(t)$ and then repeat the steps from 1 to 4 until $d(t)$ satisfies stopping criterion.

The resultant value is referred to as the first $\operatorname{IMF}$ as $\operatorname{IMF}_{1}(t)$. In order to decompose $x(t)$ into a series of IMFs the above process is repeated as follows.

(5) Subtract $\operatorname{IMF}_{1}(t)$ from $x(t)$ to generate the residual

$$
r_{1}(t)=x(t)-\operatorname{IMF}_{1}(t)
$$

(6) Treat the residual $r_{1}(t)$ as the input signal and repeat the above sifting process to generate the next $\operatorname{IMF}_{2}(t)$ and residual $r_{2}(t)$.

(7) Repeat the steps 5 and 6 to generate a series of IMFs and the last residual $r_{N}(t)$ until the stopping criterion (i.e., $r_{N}(t)$ must be monotonic) is satisfied.

Finally, the input signal can be represented as the summation of the IMFs as shown in the following equation below:

$$
x(t)=\sum_{i=1}^{N} \mathrm{IMF}_{i}(t)+r_{N}(t),
$$

where " $i$ " is the IMF order. The description of EMD in the form of a flow diagram is shown in Figure 1.

Steps 2-4 define the generation of a single IMF using sifting process and the steps 5-7 define the procedure to generate possible number of IMFs for the given signal until the stopping criterion is satisfied.

\section{Adaptive Boosting (AdaBoost)}

The AdaBoost algorithm was introduced in 1995 by Freund and Schapire [10]. A good overview is given in [11]. AdaBoost is widely used for improving the performance of any kind of learning algorithm as in [12].

AdaBoost is an efficient method which is used to increase the accuracy and robustness of the given learning algorithm. By giving a set of examples with initial weights, AdaBoost trains an initial weak learner with the given training dataset. It then focuses on those training examples which are misclassified. Then, the second weak learner is trained with an

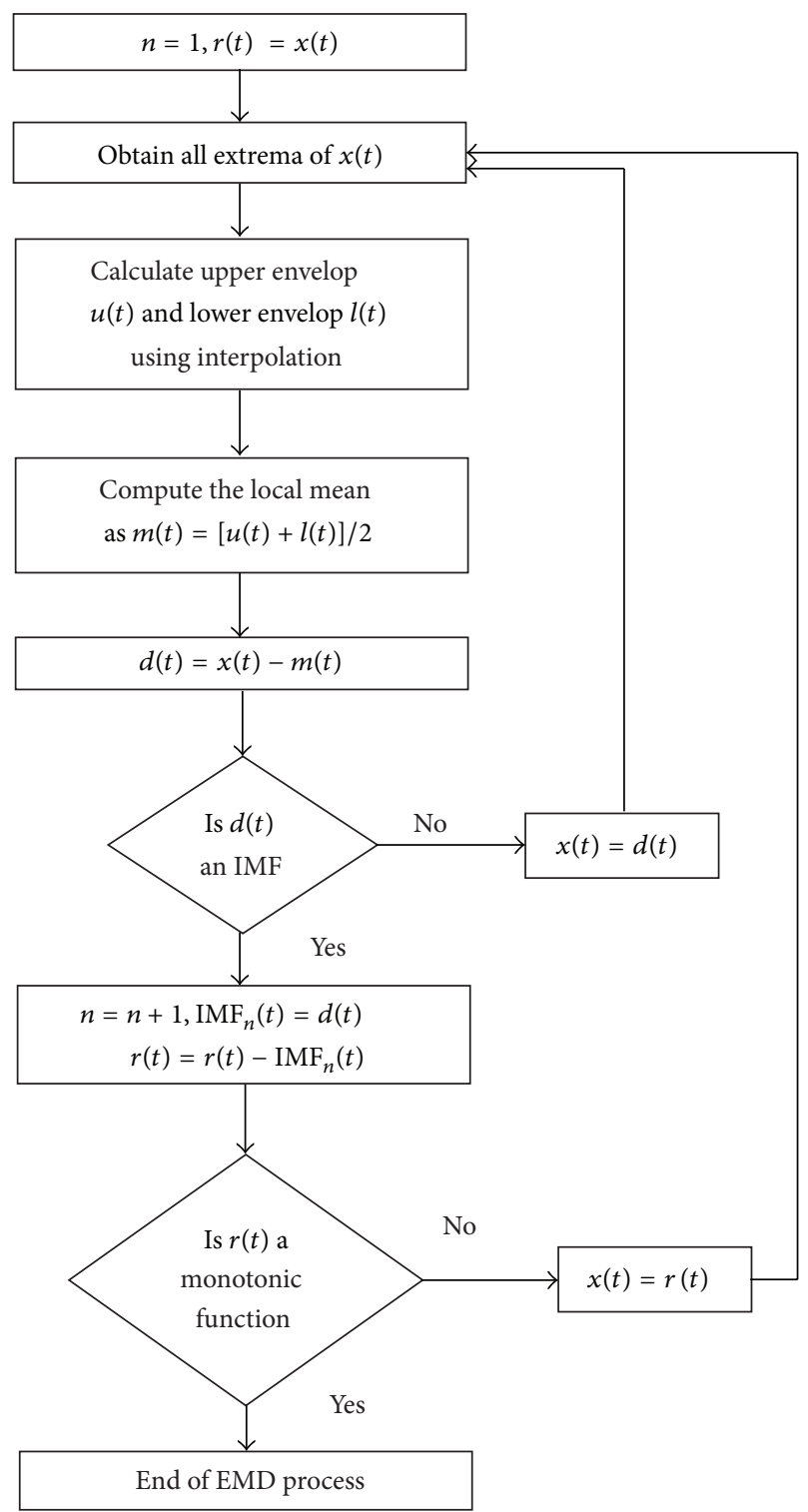

FIGURE 1: Flow diagram of EMD process.

updated training dataset which increases the weights of these misclassified examples. Finally, an ensemble is combined linearly by these trained weak learners with corresponding weights. The algorithm is described as follows.

The input data required for the AdaBoost algorithm are

$$
\text { Training set } D=\left\{\left(x_{1}, y_{1}\right), \ldots,\left(x_{L}, y_{L}\right)\right\} \text {, }
$$

where $x_{i} \in X$ (feature extracts of IMFs are applied here)

$$
y_{i} \in Y\{-1,+1\} \quad \text { (binary classification). }
$$

(1) Initially set all the sample weights as equal

$$
k_{1}(i)=\frac{1}{L}
$$

where $i=1,2, \ldots, L$. 
(2) The distribution $k_{m}$ is obtained by normalizing the weights as

$$
k_{m}(i)=\frac{k_{m}(i)}{\sum_{i=1}^{L} k_{m}(i)} .
$$

(3) Train the weak learner $h_{m}^{\text {weak }}: x \rightarrow\{-1,+1\}$ using the distribution $k_{m}$ by minimizing

$$
h_{m}=\sum_{i=1}^{L} k_{m}(i)\left\{\begin{array}{ll}
1, & \text { if } h_{m}^{\text {weak }} \\
0, & \text { else. }
\end{array}\left(x_{i}\right) \neq y_{i},\right.
$$

(4) Measure the goodness by calculating the error as

$$
e_{m}=\operatorname{Prob}_{i \sim k_{m}}\left[h_{m}^{\text {weak }}\left(x_{i}\right) \neq y_{i}\right] .
$$

(5) Calculate the $\alpha_{m}$ which is used to describe the importance assigned to $h_{m}$ as

$$
\alpha_{m}=\frac{1}{2} \ln \left(\frac{1-e_{m}}{e_{m}}\right) .
$$

(6) If $e_{m}=0$ then AdaBoost operation is to be concluded or else if $e_{m}>1 / 2$ then update the distribution as

$$
k_{m+1}(i)=k_{m}(i) \begin{cases}e^{-\alpha_{m}}, & \text { if } h_{m}^{\text {weak }}\left(x_{i}\right)=y_{i}, \\ e^{\alpha_{m}}, & \text { if } h_{m}^{\text {weak }}\left(x_{i}\right) \neq y_{i} .\end{cases}
$$

(7) Then final output hypothesis which is treated as a strong hypothesis is obtained as

$$
h^{\text {strong }}(x)=\operatorname{sign}\left(\sum_{m=1}^{T} \alpha_{m} h_{m}^{\text {weak }}(x)\right) .
$$

Here $m=1,2, \ldots, T$ that represents the number of iterations to be carried out in the AdaBoost algorithm to achieve strong hypothesis. The description of AdaBoost technique in the form of a flow diagram is shown Figure 2.

\section{Adaboost Based EMD: Proposed Method}

In this paper, a novel classification scheme for the IMFs using AdaBoost algorithm as shown in Figure 3 is proposed. The AdaBoost technique works out the operation in two stages, that is, in terms of training and testing process.

The noise corrupted signal is applied to EMD and IMFs are generated by decomposition process. In general, noise signals are treated as high frequency components. Hence earlier extracted IMFs are treated as noise-led IMFs and are discarded as like in [13]. But there is a probability of having signal contents in the discarded IMFs. Hence to adaptively select the signal-led IMFs, among the generated IMFs AdaBoost technique is used as a classifier. The generated IMFs are adaptively selected based on the features related to the clean signal.

From Figure 3 it can be observed that the algorithm is first trained by using the feature extracts of the IMFs produced by

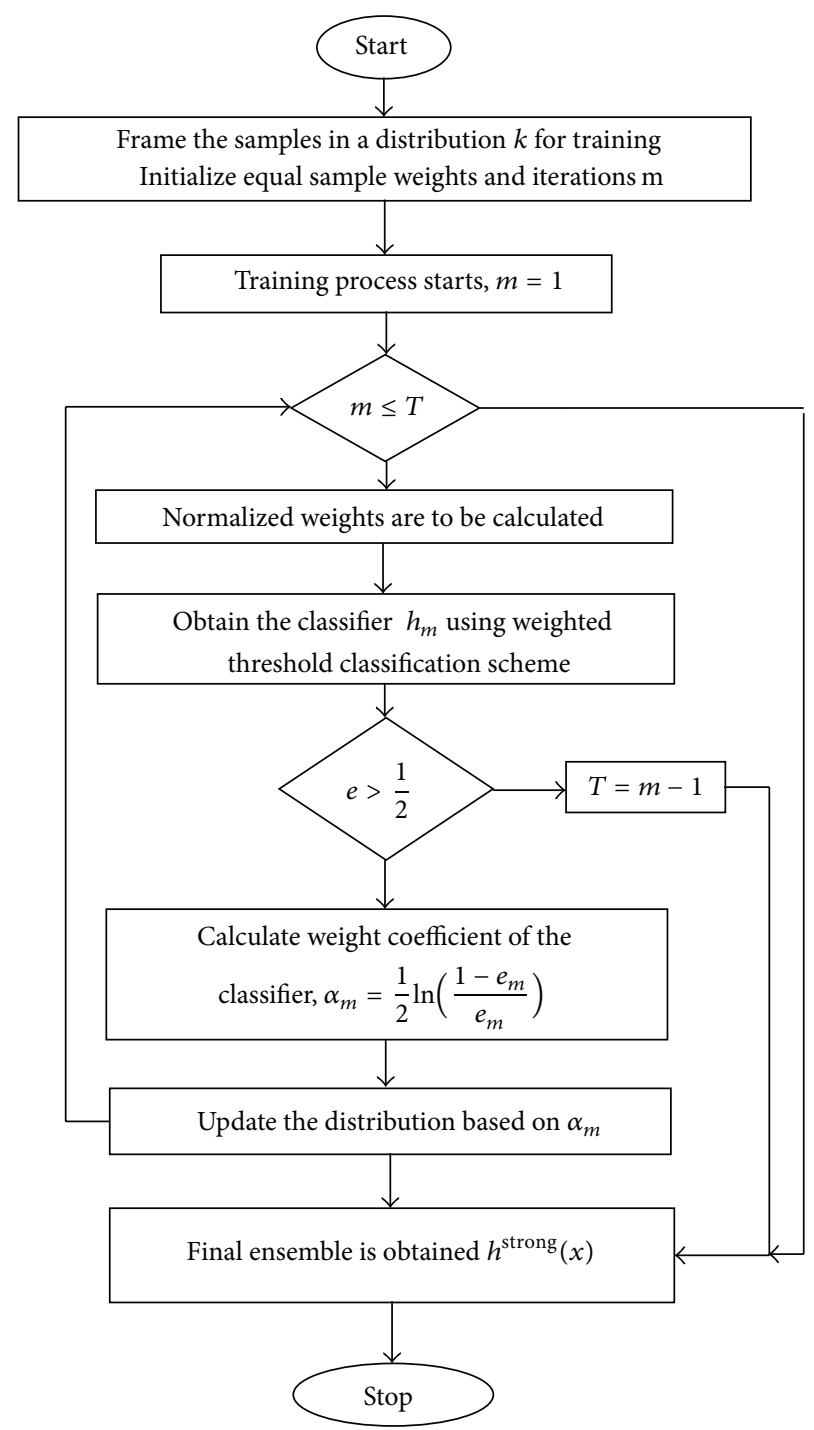

Figure 2: Flow diagram of AdaBoost Technique.

EMD for the clean signal and noise signal individually. Once the algorithm is trained, then it is used in the testing process. Again the features are calculated for all the noisy IMFs and applied to the AdaBoost algorithm in the testing process. Now the AdaBoost algorithm is capable of distinguishing the noise-led IMFs (high frequency component signals) and signal-led IMFs (low frequency component signals) and produces the ensemble of signal-led IMFs based on the feature extracts.

In this paper for the feature extraction, some of the features like mean, variance, skewness, and kurtosis [14, 15] both in frequency and time domain are calculated for all the IMFs. Binary classification scheme is used to achieve accuracy in differentiating the signal and noise IMFs in the AdaBoost algorithm. In this paper $T$ is considered as 50 and $L$ is considered as 500. To achieve more accuracy the value of $T$ may be increased but at the cost of computational complexity. 

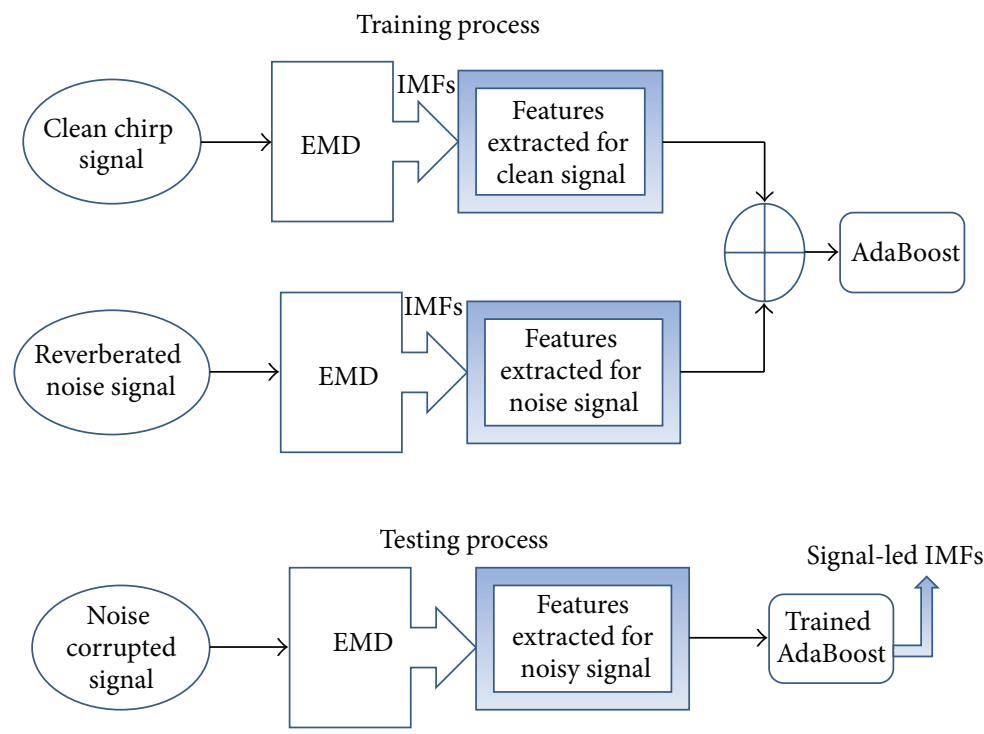

Figure 3: Process flow of EMD and AdaBoost for the selection of signal-led IMFs.

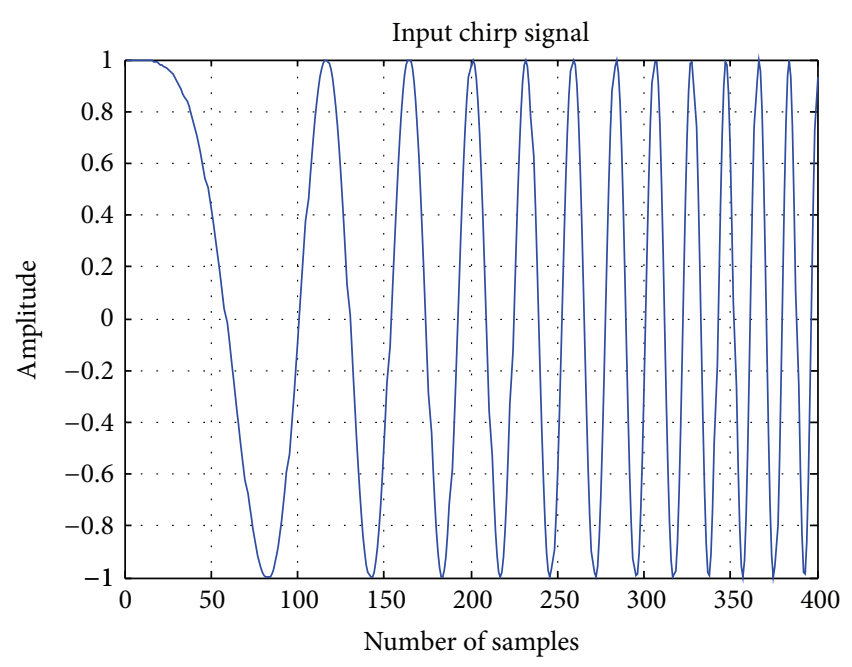

FIGURE 4: Clean chirp signal.

\section{Simulation Results}

The numerical example considered here is a chirp signal which swept from $225 \mathrm{kHz}$ to $275 \mathrm{kHz}$ over pulse duration of $100 \mu \mathrm{sec}$ spanning a bandwidth of $50 \mathrm{kHz}$, the reverberant signal is assumed to be non-stationary and colored [16]. The additive white Gaussian noise is considered as the background noise at the range of SNR's from $-10 \mathrm{~dB}$ to $10 \mathrm{~dB}$. The table and plots describe the performance and robustness in probability detection of AdaBoost based EMD. As a comparison the same process is repeated with basic EMD as dereverberation technique. The threshold value $V_{t}$ is selected as 0.5 [17].

Table 1 showed above describes the performance of AdaBoost based EMD in terms of probability detection. The plots presented here are considered for the input SNR as

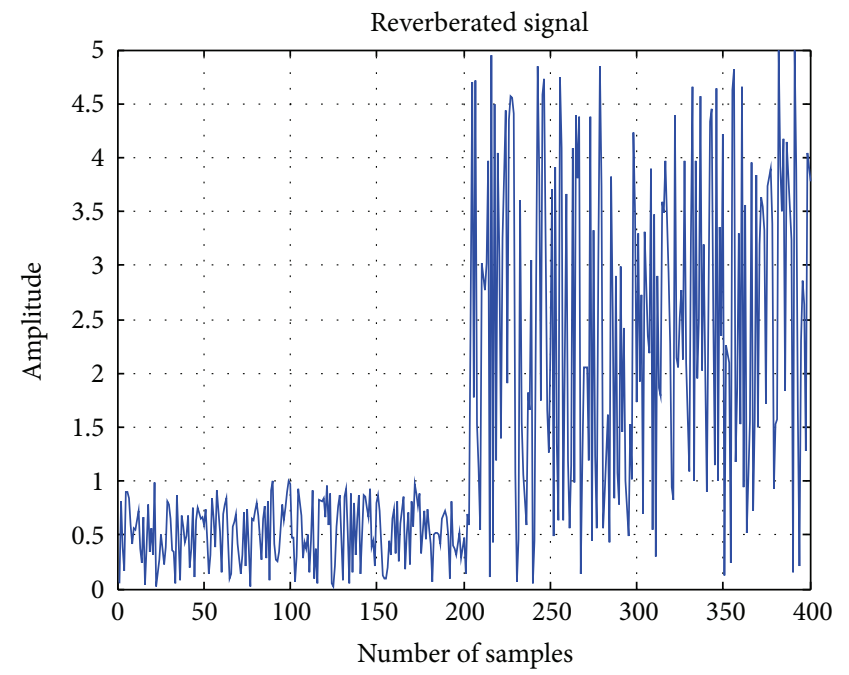

FIGURE 5: Reverberated signal.

TABLE 1: The improvement results for the range of SNR'S.

\begin{tabular}{lcc}
\hline Input SNR & \multicolumn{2}{c}{ Probability detection } \\
EMD & Ada-EMD \\
\hline-10 & 0.9050 & 0.9275 \\
-5 & 0.9075 & 0.9625 \\
-3 & 0.9575 & 0.9825 \\
0 & 0.9600 & 0.9875 \\
3 & 0.9600 & 0.9850 \\
5 & 0.9700 & 0.9875 \\
10 & 0.9450 & 0.9896 \\
\hline
\end{tabular}

$-5 \mathrm{~dB}$. Figure 4 corresponds to the input signal which is a clean chirp signal. Figure 5 describes the plot details of reverberated signal. Figure 6 describes the plot details of 


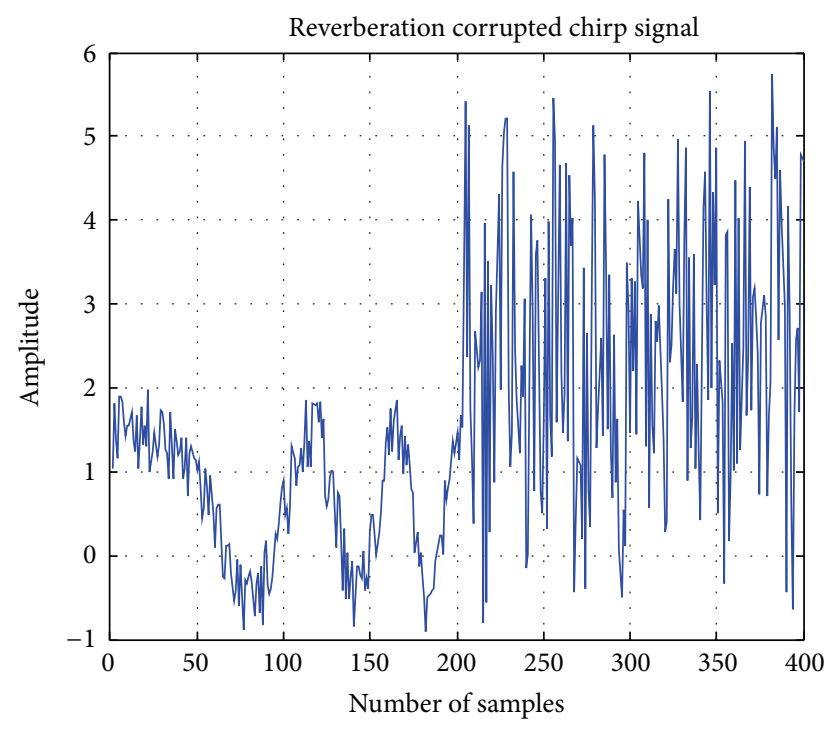

FIGURE 6: Reverberation corrupted Chirp signal.

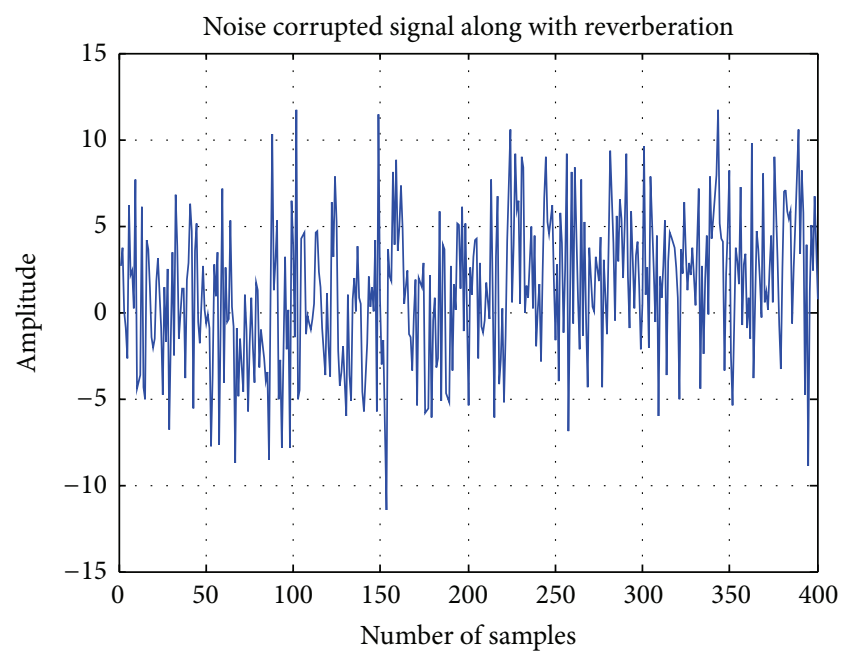

FIGURE 7: Noisy Chirp along with reverberation.

reverberation corrupted chirp signal. Figure 7 is the noise corrupted chirp signal along with reverberation.

Figure 8 provides the details of simulation plots obtained using EMD only, that is, denoising is done with basic EMD. In this plot the estimated signal is obtained by manual selection of IMFs. Figure 9 describes the simulation plots in which the estimated signal is obtained by combining the IMFs that are adaptively selected by AdaBoost, that is, based on the features, the signal-led IMFs, and noise-led IMFs are separated by the AdaBoost algorithm.

In the training process the total IMFs produced for the clean chirp signal are 4 , the IMFs produced for reverberated noise signal are 8 , and in the testing process the IMFs produced by EMD are 9. The selected IMFs by AdaBoost technique are 2, 3, 7, 8, and 9 that are used for reconstruction of the chirp signal. Figure 10 describes the probability detection
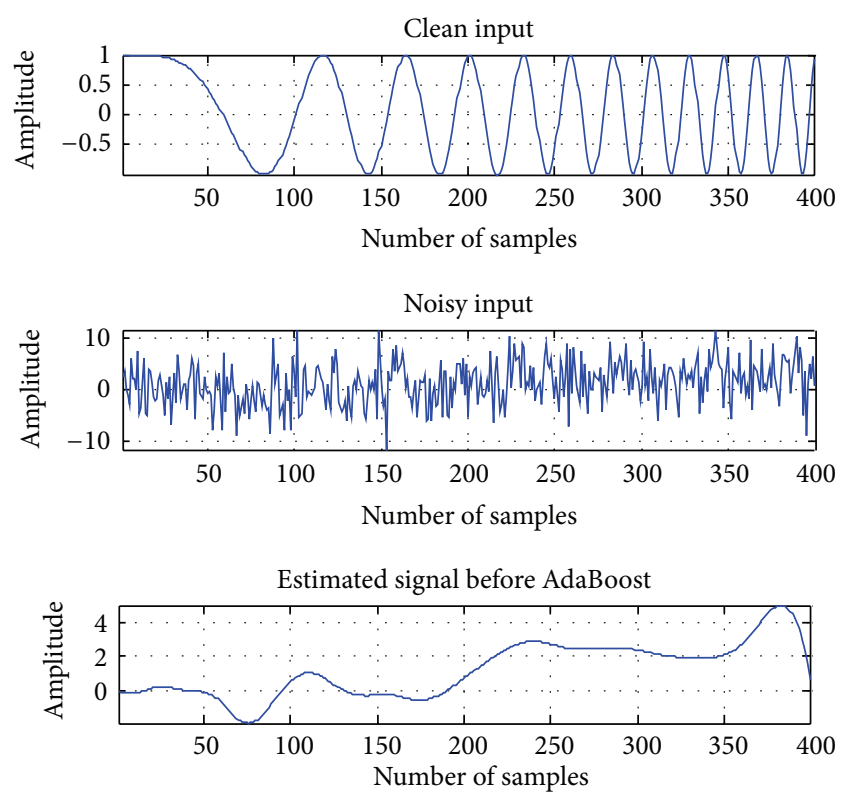

FIgURE 8: Signal detection using EMD only.
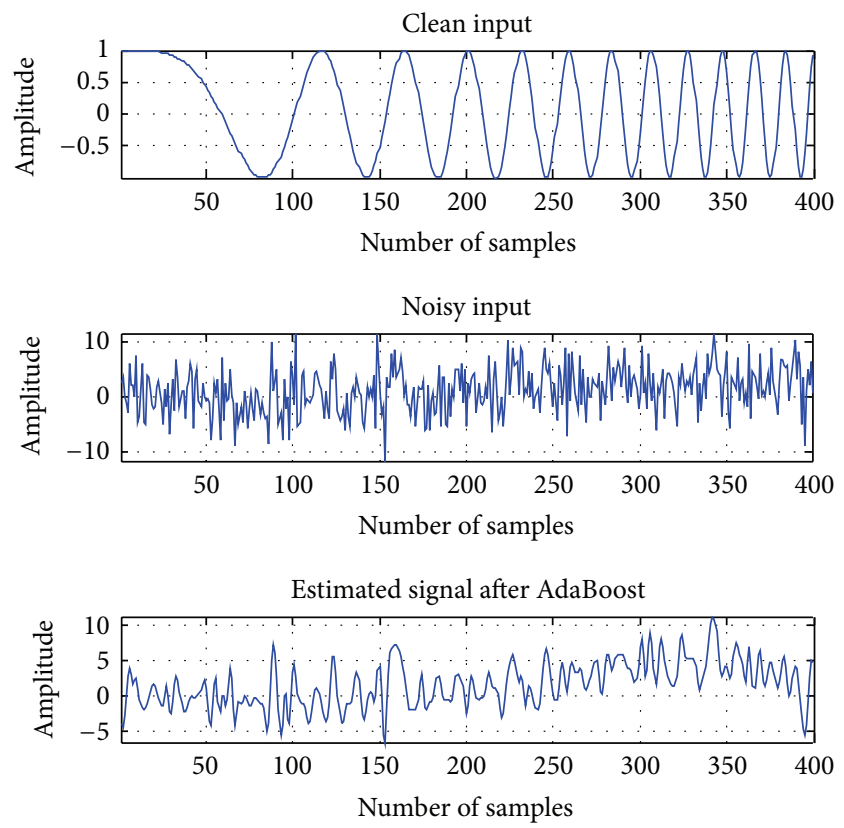

FIgURE 9: Signal detection using AdaBoost based EMD.

curves for both EMD and AdaBoost based EMD as denoising schemes. It can be observed from the plots that there is an improvement in the detection probability after filtering with AdaBoost based EMD technique.

From the plots it is evident that the reconstruction of the chirp signal even at low input SNR conditions is achieved with the use of AdaBoost based EMD as a de-noising technique. Hence this method can be treated as one of the 


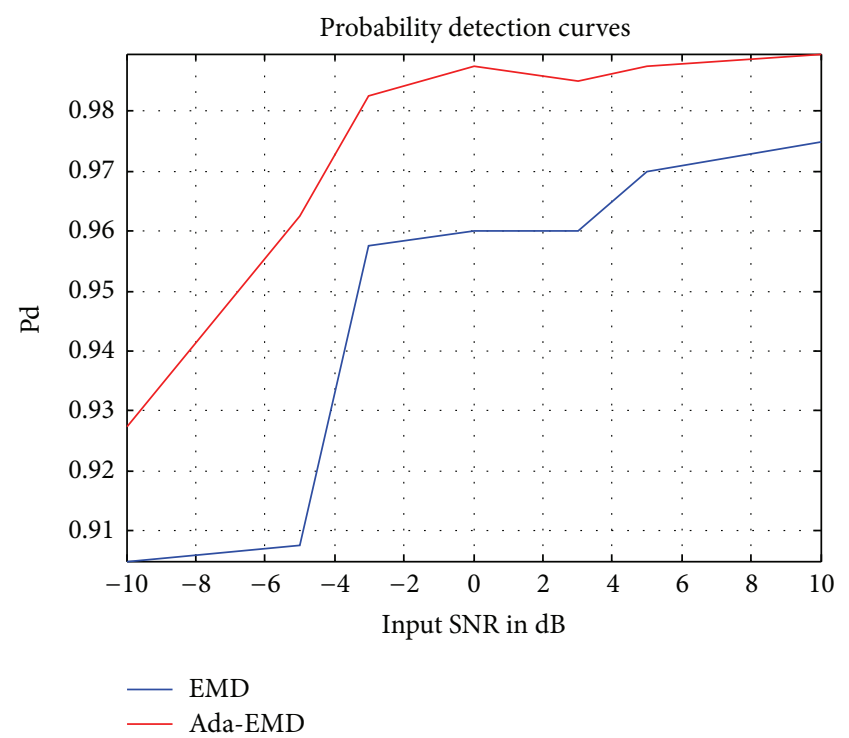

FIGURE 10: Improvement of probability detection after filtering with the AdaBoost based EMD.

best method that can be used for the joint suppression of the reverberation and the background noise effects.

\section{Conclusion}

This paper proposes AdaBoost based EMD as a new detection scheme that is capable of suppressing the combined effect of background noise and reverberation in room acoustics and in underwater environment. The performance of this detection scheme was examined by computer generated white Gaussian noise and reverberant signal.

The numerical example considered in this paper describes the effectiveness of AdaBoost based EMD in improving the probability of detection at various SNR levels. Specifically in the underwater scenario, even at low SNRs, that is, at an input SNR of $-5 \mathrm{~dB}$, the AdaBoost based EMD is capable of detecting the signal. It can be observed from the simulation plots and a probability detection curve that AdaBoost based EMD has suppressed the joint effect of background noise and reverberation more efficiently than the basic EMD method.

AdaBoost based EMD technique in combination with various parameter estimation methods can be used to improve the performance of the direction of arrival (DOA) calculation for sound signals in SONAR application which is the future scope of this paper.

\section{Conflict of Interests}

The authors declare that there is no conflict of interests regarding the publication of this paper.

\section{Acknowledgment}

This work is being supported under the project "Robust Signal Processing Techniques for RADAR/SONAR Communications using VLSI Tools" by Ministry of Science \&
Technology, Department of Science \& Technology (DST), New Delhi, India, under Women Scientist Scheme (WOS-A).

\section{References}

[1] W. Li, Q. Zhang, X. Ma, and C. Hou, "Active sonar detection in reverberation via signal subspace extraction algorithm," Eurasip Journal on Wireless Communications and Networking, vol. 2010, Article ID 981045, 2010.

[2] H. Song, P. Roux, T. Akal et al., "Time reversal ocean acoustic experiments at $3.5 \mathrm{kHz}$ : applications to active sonar and undersea communications," in Proceedings of the High Frequency Ocean Acoustics Conference, vol. 728 of AIP Conference Proceedings, pp. 522-529.

[3] N. E. Huang, Z. Shen, S. R. Long et al., "The empirical mode decomposition and the Hubert spectrum for nonlinear and non-stationary time series analysis," Proceedings of the Royal Society A, vol. 454, no. 1971, pp. 903-995, 1998.

[4] H. Ying, H. Hong, L. Li, W. Yi, and P. Hongyan, “The applications and simulation of adaptive filter in noise canceling," in Proceedings of the International Conference on Computer Science and Software Engineering (CSSE '08), pp. 1-4, December 2008.

[5] V. UdayShankar, Modern Digital Signal Processing, PHI, 2nd edition, 2012.

[6] D. L. Donoho and J. M. Johnstone, "Ideal spatial adaptation by wavelet shrinkage," Biometrika, vol. 81, no. 3, pp. 425-455, 1994.

[7] D. L. Donoho, "De-noising by soft-thresholding," IEEE Transactions on Information Theory, vol. 41, no. 3, pp. 613-627, 1995.

[8] Ch. Kusma Kumari and K. Raja Rajeshwari, "Application of EMD as a robust adaptive signal processing technique in radar/sonar communications," International Journal of Engineering Science and Technology (IJEST), vol. 3, no. 12, pp. 82628266, 2011.

[9] Kusma Kumari Ch and K. Raja Rajeswari, "Enhancement of performance measures using EMD in noise reduction application," International Journal of Computer Applications, vol. 70, no. 5, pp. 10-14, 2013.

[10] Y. Freund and R. E. Schapire, "A decision-theoretic generalization of on-line learning and an application to boosting," Journal of Computer and System Sciences, vol. 55, no. 1, pp. 119-139, 1997.

[11] Y. Freund and R. Schapire, "A short introduction to boosting," Journal of Japanese Society For Artificial Intelligence, vol. 14, no. 5, pp. 771-780, 1999.

[12] R. Schapire, “The boosting approach to machine learning: an overview," in Proceedings of the MSRI Workshop on Nonlinear Estimation and Classification, 2001.

[13] W.-B. Jin and Y. Jun, "Velocity determination of single sound source based on Doppler Effect," in Proceedings of the International Conference on Wavelet Analysis and Pattern Recognition (ICWAPR '07), pp. 956-961, Beijing, China, November 2007.

[14] A. Kumar, P. Agarwal, P. Dighe, S. S. Bhiksha Raj, and K. Prahallad, "Speech Emotion Recognition by AdaBoost Algorithm and Feature Selection for Support Vector Machines," http://home .iitk.ac.in/ subhali/reports/report_iptse.pdf.

[15] “Speech Feature Tool," Centre for Speech Technology University of Edinburgh, http://festvox.org/docs/speech_tools-1.2.0/c23 .htm.

[16] G. Ginolhac, J. Chanussot, and C. Hory, "Morphological and statistical approaches to improve detection in the presence of 
reverberation," IEEE Journal of Oceanic Engineering, vol. 30, no. 4, pp. 881-899, 2005.

[17] A. Burguera, G. Oliver, and Y. González, "Range extraction from underwater imaging sonar data," in Proceedings of the 15th IEEE International Conference on Emerging Technologies and Factory Automation (ETFA '10), September 2010. 

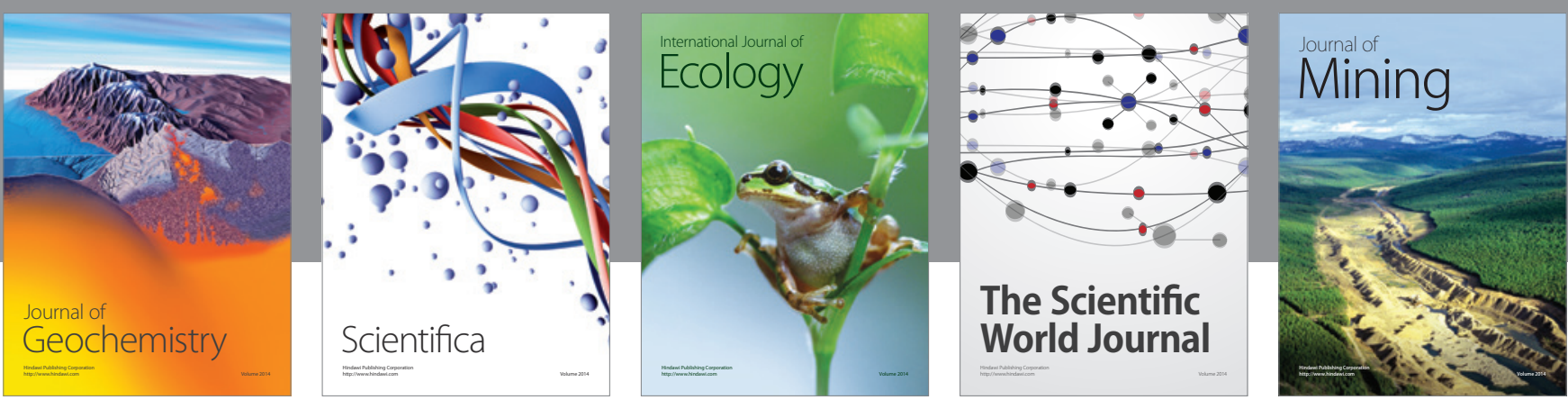

The Scientific World Journal
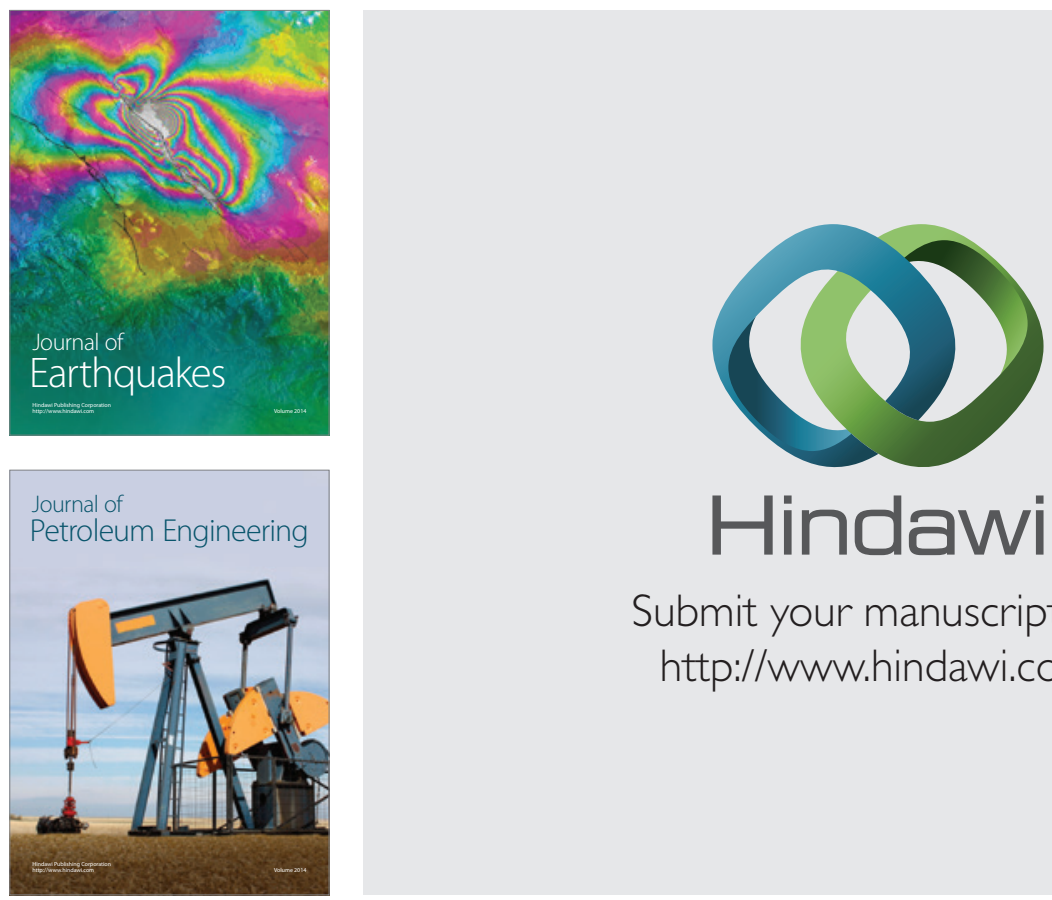

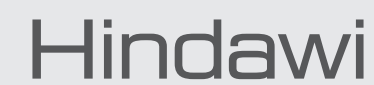

Submit your manuscripts at

http://www.hindawi.com
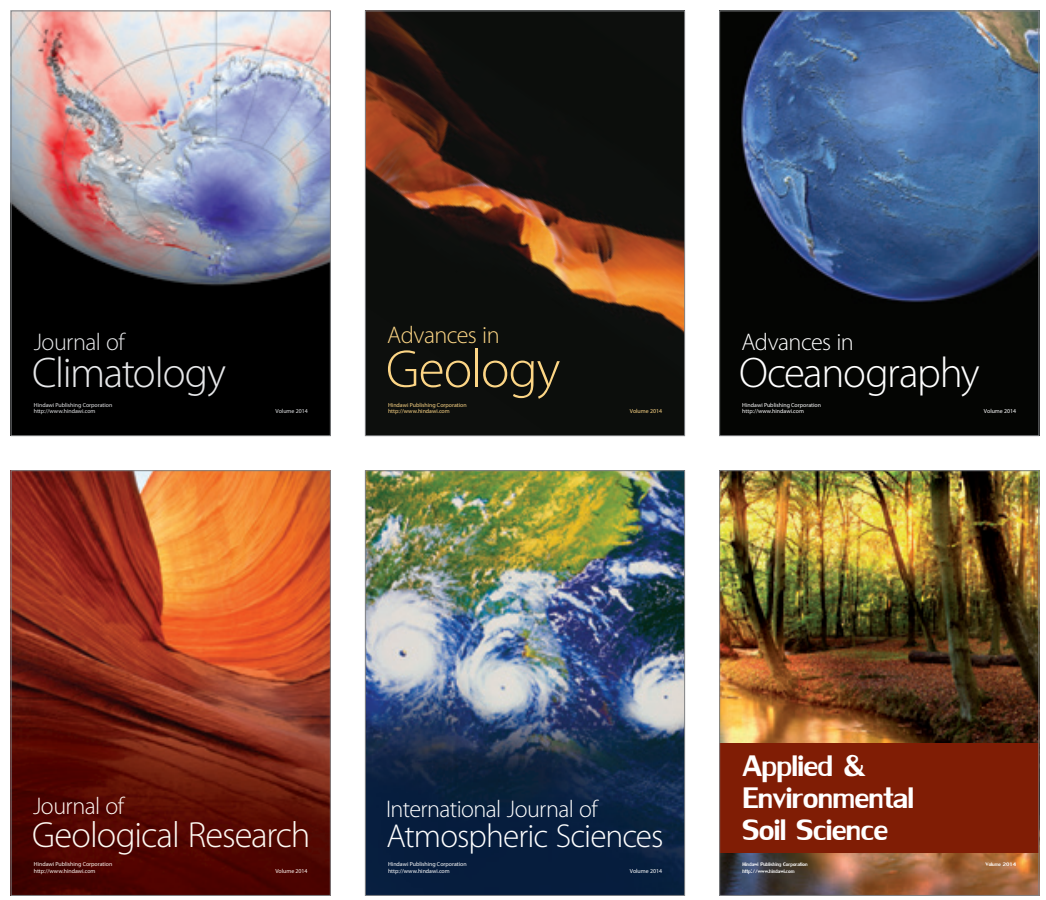
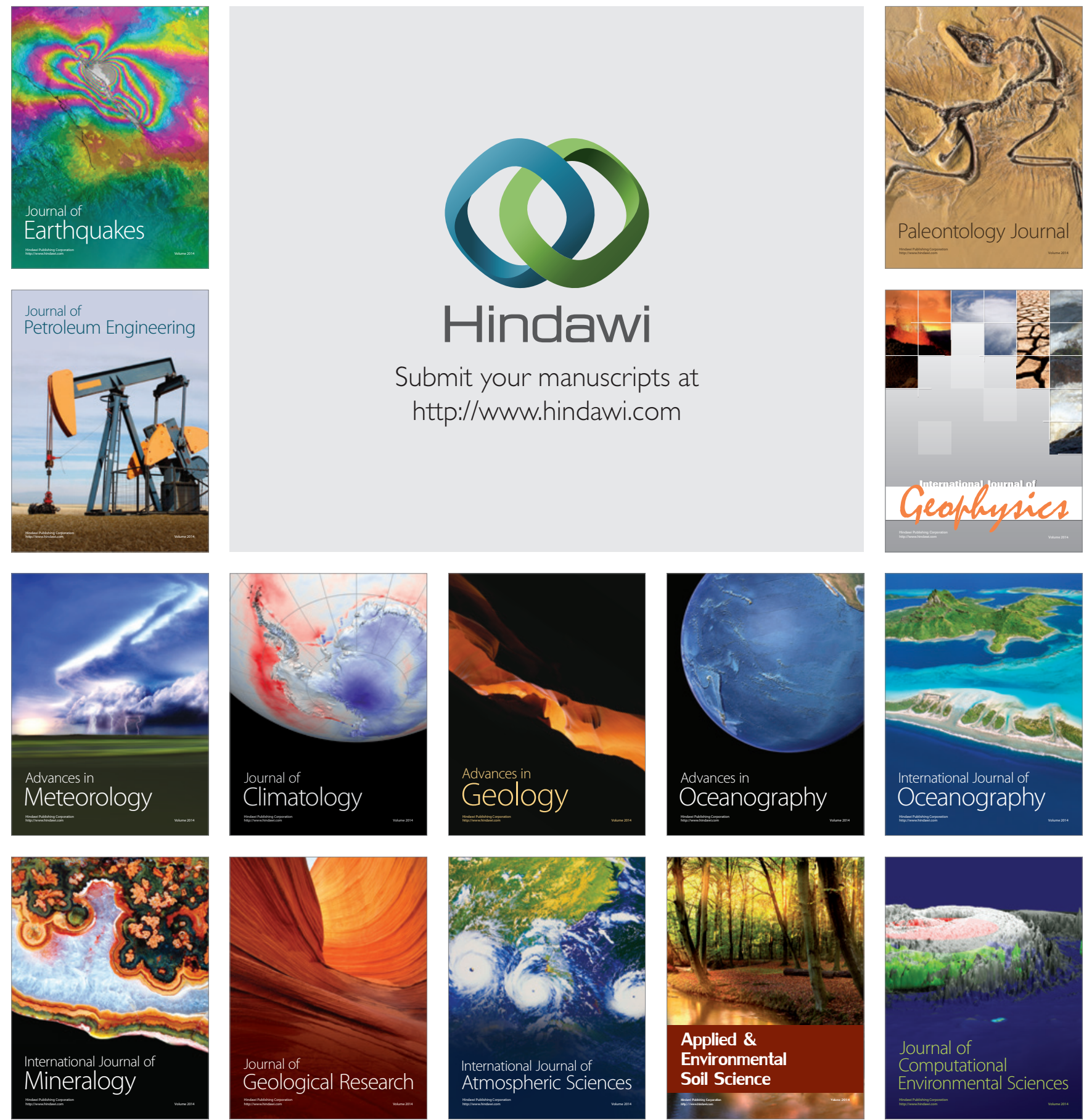